University of Nebraska - Lincoln

DigitalCommons@University of Nebraska - Lincoln

$1-1-1987$

\title{
Nonorthogonal configuration-interaction calculations of molecular states: The low-lying molecular states of ArHsup+ and two-electron charge exchange in $\mathrm{Ar}-\mathrm{H}^{+}$collisions
}

Gordon A. Gallup

University of Nebraska-Lincoln, ggallup1@unl.edu

Follow this and additional works at: https://digitalcommons.unl.edu/physicsgallup

Part of the Physics Commons

Gallup, Gordon A., "Nonorthogonal configuration-interaction calculations of molecular states: The lowlying molecular states of ArHsup+ and two-electron charge exchange in $\mathrm{Ar}-\mathrm{H}^{+}$collisions" (1987). Gordon Gallup Publications. 7.

https://digitalcommons.unl.edu/physicsgallup/7

This Article is brought to you for free and open access by the Research Papers in Physics and Astronomy at DigitalCommons@University of Nebraska - Lincoln. It has been accepted for inclusion in Gordon Gallup Publications by an authorized administrator of DigitalCommons@University of Nebraska - Lincoln. 


\title{
Physical ReView A
}

\author{
GENERAL PHYSICS
}

\section{Nonorthogonal configuration-interaction calculations of molecular states: The low-lying molecular states of $\mathrm{ArH}^{+}$and two-electron charge exchange in $\mathrm{Ar}-\mathrm{H}^{+}$collisions}

\author{
G. A. Gallup \\ Department of Chemistry, University of Nebraska, Lincoln, Nebraska 68588-0304
}

(Received 23 June 1986)

\begin{abstract}
A nonorthogonal configuration-interaction procedure has been used to calculate adiabatic energy curves of $\mathrm{ArH}^{+}$. The basis functions are a mixture of typical molecular-orbital configurations and typical valence-bond (Heitler-London) configurations. This gives an economical description of the wave functions, allowing an easy tracing of various diabatic states through the adiabatic states and a clear identification of the asymptotic states. The results are used to rationalize the unexpectedly large production of $\mathrm{Ar}^{2+}+\mathrm{H}^{-}$from collisions of $\mathrm{H}^{+}$with $\mathrm{Ar}$.
\end{abstract}

\section{INTRODUCTION}

Experiments determining the two-electron chargetransfer (2ECT) cross sections during collisions of protons on rare-gas atoms show unexpectedly large values. ${ }^{1-3}$ The results from different laboratories agree well, and for the particular case of $\mathrm{H}^{+}+\mathrm{Ar}$ the cross section for the production of $\mathrm{H}^{-}+\mathrm{Ar}^{2+}$ averages $(2-3) \times 10^{-17} \mathrm{~cm}^{2}$ over the energy range of $2-50 \mathrm{keV}$. More recently, Van Zyl, Rothwell, and Neumann, ${ }^{4}$ studying collisions between protons and Ar, have determined the cross sections for the production of excited $\mathbf{H}$ atoms during charge transfer. To interpret their data they consider it essential that the twoelectron charge-transfer process be included in the analysis of the various coupled states during the reaction. Experiments involving collisions of $\mathrm{H}^{+}$with other raregas atoms show similar effects. ${ }^{5}$

This article describes calculations of some of the lowlying molecular energy curves of $\mathrm{ArH}^{+}$to help understand 2ECT during the collisions. A nonorthogonal configuration-interaction (NCI) method is used. A key feature of this procedure is the mixing of molecularorbital configurations with valence-bond (Heitler-London) configurations in the basis. ${ }^{6}$ This provides an economical representation of a large number of the energy states of the molecule and a clear picture of the asymptotic character of the states at large nuclear separations. Although we treat only $\mathrm{ArH}^{+}$in this article, the calculations should also provide a model of the interactions for the other rare-gas atoms.

The first two ionization potentials (IP) of Ar are 15.755 and $27.62 \mathrm{eV}$, the IP of $\mathrm{H}$ is $13.605 \mathrm{eV}$, and the electron affinity of $\mathrm{H}$ is $0.75 \mathrm{eV}$. Thus the state $\mathrm{Ar}^{2+}\left({ }^{1} D\right)+\mathrm{H}^{-}$is $15.00 \mathrm{eV}$ above the onset of the one-electron continuum, $\mathrm{Ar}^{+}\left({ }^{2} \mathrm{P}\right)+\mathrm{H}^{+}+e^{-}$, and a calculation with discrete basis functions clearly cannot duplicate the infinite number of Rydberg states and portions of the continuum occurring in this energy range. Nevertheless, the valence-bond configurations present in the basis give a good account of the corresponding asymptotic states, and other basis functions combine to give eigenfunctions that are representative of Rydberg or continuum states in the same energy region. This provides information for arguments we make about the interaction of the $\mathrm{Ar}^{2+}+\mathrm{H}^{-}$state with Rydberg and continuum states.

In its simplest terms one may formulate the configuration-interaction (CI) problem using Slater determinantal functions (SDF) for the $n$-electron basis. If the spin orbitals used in the SDF are all orthonormal, the CI is said to be orthogonal. This general scheme for electronic structure calculations of molecules has been efficiently implemented during the past few years. ${ }^{7,8}$ Nevertheless, the constraints implicit and explicit in the orthonormal set of spin orbitals required to produce an orthogonal CI make it an awkward method for describing some sorts of physical situations. A particularly troublesome example is the kind of situation we treat here where we wish to discuss excited states of a dissociating system.

For dissociating systems, a basis that goes naturally over into simple representations of the asymptotic states is conceptually the most useful. This leads to generalizations of the Heitler-London or valence-bond method and necessarily involves spin orbitals that are nonorthogonal and a NCI procedure. Of course, once one implements a method that abandons the orthonormality constraint on the orbitals or spin orbitals, one has complete freedom thereafter to mix orbitals in any way so that the physical content of the wave function best describes the actual system. Later, we indicate how this freedom allows an economical description of the wave functions for $\mathrm{ArH}^{+}$. 
In this paper we first give a detailed description of our NCI procedure. For a different method see Raimondi et al. ${ }^{9}$ The outline of the method is followed by an account of the specific details of this calculation and we end with a discussion of the formation and probable fate of the $\mathrm{Ar}^{2+}+\mathrm{H}^{-}$state during the collision.

\section{NONORTHOGONAL CONFIGURATION-INTERACTION CALCULATIONS}

Löwdin ${ }^{10}$ gave the first discussion of a general method for evaluating the matrix elements of the Hamiltonian between nonorthogonal functions. We write the nonrelativistic Hamiltonian in the standard form

$$
H=E_{\text {nuc }}+F+G,
$$

where

$$
\begin{aligned}
& E_{\mathrm{nuc}}=\sum_{\substack{\alpha, \beta \\
(\text { alpha }<\beta)}} Z_{\alpha} Z_{\beta} / r_{\alpha \beta}, \\
& F=\sum_{i} f_{i}=\sum_{i}-\frac{1}{2} \nabla_{i}^{2}+\sum_{i, \alpha} Z_{\alpha} / r_{\alpha i} \\
& G=\sum_{\substack{i, j \\
(i<j)}} 1 / r_{i j}
\end{aligned}
$$

Löwdin showed that the matrix element of the Hamiltonian (1) between two SDF's of nonorthogonal spin orbitals can be written in terms of first- and second-order cofactors of the determinant of the matrix of overlaps between the spin orbitals. Thus if we have

$$
\Psi=N_{u}\left|u_{1} u_{2} \cdots u_{n}\right|
$$

and

$$
\Phi=N_{v}\left|v_{1} v_{2} \cdots v_{n}\right|
$$

as the two SDF's, we define the matrix of the overlaps between the two sets of spin orbitals as

$$
\left(S_{u v}\right)_{i j}=\left\langle u_{i} \mid v_{j}\right\rangle \text {. }
$$

The formula for the matrix element $\langle\Psi|H| \Phi\rangle$ may now be written as

$$
\begin{aligned}
& \langle\Psi|H| \Phi\rangle
\end{aligned}
$$

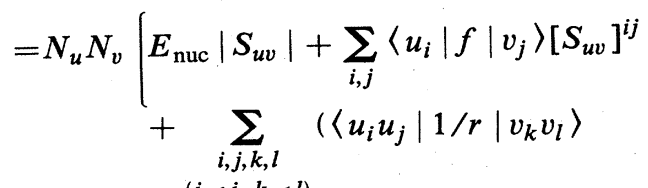

$$
\begin{aligned}
& (i<j, k<l) \\
& \left.\left.-\left\langle u_{i} u_{j}|1 / r| v_{l} v_{k}\right\rangle\right)\left[S_{u v}\right]^{i j, k l}\right] .
\end{aligned}
$$

where the one- and two-electron integrals have their conventional meanings, $\left|S_{u v}\right|$ is the determinant of the overlaps, $\left[S_{u v}\right]^{i j}$ is the cofactor (signed minor) of the $i j$ element of $\left|S_{u v}\right|$, and $\left[S_{u v}\right]^{i j, k l}$ is the cofactor of the $i j-k l$ $(i<j, k<l) 2 \times 2$ minor of $\left|S_{u v}\right|$.

The direct evaluation of the cofactors, by Cramer's rule, for example, is very time consuming and precludes using such a scheme in (5) for a practical calculation of any size. An essential contribution made by Löwdin was his observation that if $\left|S_{u v}\right| \neq 0$ the cofactors in (5) may be obtained much more economically by first calculating $S_{u v}^{-1}$ and using the formulas ${ }^{11}$

$$
\begin{aligned}
& {\left[S_{u v}\right]^{i j}=\left|S_{u v}\right|\left(S_{u v}^{-1}\right)_{j i},} \\
& {\left[S_{u v}\right]^{i j, k l}=\left|S_{u v}\right|\left\{\left(S_{u v}^{-1}\right)_{i k}\left(S_{u v}^{-1}\right)_{j l}-\left(S_{u v}^{-1}\right)_{i l}\left(S_{u v}^{-1}\right)_{i k}\right\} .}
\end{aligned}
$$

In these times when computers are used for extensive calculations, it is customary to characterize algorithms as requiring polynomial or exponential times. Furthermore, if we have a polynomial time algorithm, it will have a certain degree, $n^{k}$, indicating how rapidly the time for a calculation increases as the number of items (in this case the number of electrons) dealt with increases.

It is important to appreciate why the use of (6) and (7) represents such a savings in effort over the direct evaluation of determinants in (5). We note that Gaussian elimination used either for evaluating determinants or for matrix inversion is an $n^{3}$ algorithm. The most timeconsuming portion of (5) to calculate is the last summation, which involves $n^{2}(n-1)^{2} / 4$ terms and hence requires, at best, an $n^{4}$ algorithm for evaluation. If the method of Gaussian elimination is applied separately to obtain each $\left[S_{u v}\right]^{i j, k l}$, the total time to evaluate the twoelectron part of the matrix element is proportional to $n^{7}$. The time savings obtained using (6) and (7) arise because $S_{u v}^{-1}$ needs to be calculated only once for each matrix element, and then each cofactor may be obtained from the elements of $S_{u v}^{-1}$ in a time independent of $n$.

As a result of this analysis, we see that, unless some method is devised for simplifying the last summation in (5), the best we can do in evaluating the matrix elements of nonorthogonal SDF's is an $n^{4}$ algorithm. Of course, if the rank of the matrix $S_{u v}$ is less than $n-2$, the determinant and all of the cofactors in (5) are zero. If this condition occurs often enough to be important, an $n^{3}$ (or faster) method for detecting it can speed up computations.

The formulas (6) and (7) break down if $S_{u v}$ is a singular matrix, and the progress made in dealing with nonorthogonal configurations since Löwdin's original contributions has been in dealing with such cases. As pointed out in the last paragraph, one need treat singular cases only if the $\operatorname{rank}\left(S_{u v}\right)=(n-1)$ or $(n-2)$. King et al. ${ }^{12}$ give a method for calculating the cofactors that first requires a diagonalization of the matrix $S_{u v}$. Later, Prosser and Hagstrom ${ }^{13}$ show how cofactors can be calculated from a purely finite algebraic biorthogonalization procedure. This latter method is an $n^{3}$ algorithm. More recently Norbeck and Gallup ${ }^{14}$ gave another $n^{3}$ algorithm for obtaining the necessary cofactors. This method is distinguished from the others in that, when $\left|S_{u v}\right|=0$, the matrix is modified to be nonsingular. Therefore, the Gaussian elimination method may still be used to evaluate the inverse in a form such that the cofactors may be extracted from it in times independent of $n$. The method of Ref. 14 has been simplified since 1973, and the presently used version is given by Gallup et al. ${ }^{15}$

It should be understood from the previous discussion 
that the methods available for evaluating the cofactors in (5) do not add to the exponent of $n$ in the polynomial time of the total algorithm for obtaining the matrix element. ${ }^{16}$ If it must be calculated at all, it requires an $n^{4}$ algorithm.

Actually, the ease of evaluation of matrix elements between two SDF's is only part of the problem. To make calculations more efficient and more practical it is desirable to use functions in a CI calculation that have specific spin and spatial symmetry properties. We take up the problem of the spin properties first.

It is well known that single SDF's are not eigenfunctions of the total spin operator unless all of the unpaired electrons are described by spin orbitals with the same $m_{s}$ value. Otherwise, linear combinations of SDF's involving permuted spin functions among the orbitals are required. If we have a set of SDF's with $q$ unpaired electrons and differing only in the arrangements of the spin functions among the orbitals, the number of linearly independent functions, $n_{f}$, with a projection quantum number $M_{s}$ is the binomial coefficient

$$
n_{f}=\left(\begin{array}{c}
q \\
q / 2-M_{s}
\end{array}\right) \text {. }
$$

An eigenfunction of the spin with total spin quantum number $S \geq\left|M_{S}\right|$ is a linear combination of these $n_{f}$ SDF's, and there are $n_{S}$ linearly independent eigenfunctions belonging to $S$, where

$$
n_{S}=(2 S+1)\left(\begin{array}{c}
q \\
q / 2-S
\end{array}\right) /(q / 2-S+1) .
$$

One particular set of linear combinations that form eigenfunctions of $S^{2}$ can be constructed using the spin projection operators originally devised by Löwdin, ${ }^{17}$

$$
\Psi_{S}=O_{S} N_{u}\left|u_{1} u_{2} \cdots u_{n}\right| \text {. }
$$

Pauncz ${ }^{18}$ has given a complete discussion of the construction of spin eigenfunctions. Gallup ${ }^{19}$ has shown that the type of functions generated in (10) form a basis for the Young's natural representation of the symmetric groups. ${ }^{20}$ Using the theory of representation of the symmetric groups, a somewhat lengthy argument, ${ }^{21}$ which we do not repeat here, then allows one to recast the matrix elements $\left\langle\Psi_{S}|H| \Phi_{S}\right\rangle$ in terms of determinantal functions different in type from SDF's. The savings here is that, in most cases, considerably fewer than $n_{f}$ terms are required to generate matrix elements for pure spin states.

Constructing states of pure spatial symmetry can also result in a savings of effort in evaluating the matrix of the Hamiltonian, if not all symmetries of states are to be treated. In our procedure we accomplish this by projecting out the desired states before the matrix diagonalizations. $^{22}$ This is taken care of automatically by the programs used in this study, and we do not discuss this further at this time.

\section{DETAILS OF CALCULATION}

Some years ago Vance and Gallup ${ }^{23}$ devised a basis, including Rydberg orbitals on $\mathrm{Ar}$ and $\mathbf{H}$, for a theoretical study of the quenching of excited Ar atoms by collisions
TABLE I. Comparison of selected energy differences (in eV).

\begin{tabular}{lccc}
\hline \hline & SCF & NCI & Expt. \\
\hline$D_{e}$ & $2.96 \mathrm{eV}$ & $3.34 \mathrm{eV}$ & $2.6-4.16^{\mathrm{a}} \mathrm{eV}$ \\
& & & \\
& Asymptotic energies & \\
$\mathrm{Ar}^{2+}\left({ }^{1} D\right)+\mathrm{H}^{-}$ & & 33.75 & 30.76 \\
$\mathrm{Ar}^{2}\left(4 s^{2} P\right)+\mathrm{H}^{+}$ & & 11.84 & 11.70 \\
$\mathrm{Ar}+\mathrm{H}$ & & 2.66 & 2.15 \\
$\mathrm{Ar}+\mathrm{H}^{+}$ & & 0.00 & 0.00 \\
\hline \hline
\end{tabular}

${ }^{a}$ Considerable variance exists among various experimental and theoretical determinations of $D_{e}$. See K. P. Huber and G. Herzberg, Constants of Diatomic Molecules (Van Nostrand Reinhold, New York, 1979), and P. Rosmus, Theor. Chim. Acta 51, 359 (1979), and references therein.

with $\mathrm{H}$ atoms. The same basis was used for the present studies on $\mathrm{Ar}+\mathrm{H}^{+}$, and the reader is referred to the earlier paper for the details.

The molecular configuration of $\mathrm{ArH}^{+}$, $(1 \sigma)^{2}(2 \sigma)^{2}(3 \sigma)^{2}(4 \sigma)^{2}(5 \sigma)^{2}(1 \pi)^{4}(2 \pi)^{4}$, is the same at all internuclear distances, and the self-consistent-field (SCF) wave function smoothly dissociates in the ground state. Table I shows the SCF value of $D_{e}$ along with a number of other quantities from the present calculation.

As was pointed out above, we use orbitals from both the molecular SCF calculation and the original atomic SCF calculations in constructing the $n$-electron functions for this NCI calculation. All of the configurations had the "core" electrons, $1 \sigma, 2 \sigma, 3 \sigma$ and $1 \pi$, doubly occupied in every case. The list of orbitals used appears in Table II. The $\mathrm{H}$ orbital designated $1 s^{\prime}$ is the only one that is not self-explanatory. It is a five-Gaussian approximation to a is $\mathrm{H}$ orbital with the effective charge set to 0.3 . This is very close to the optimum value in a "split orbital" calculation of the $\mathrm{H}^{-}$ion. ${ }^{24}$ As we shall see later, its presence in the basis allows a flexible description of $\mathrm{H}^{-}$as the internuclear separation changes.

The configurations selected were chosen by criteria based in part on the physical nature of the states to be described and in part on convenience. They fall into four classes.

(1) The principal configuration (core) $(4 \sigma)^{2}(5 \sigma)^{2}(2 \pi)^{4}$.

(2) All single excitations from the four active orbitals into all of the other available orbitals.

TABLE II. Orbitals used in NCI calculation.

\begin{tabular}{clc}
\hline \hline Molecule & Ar & $\mathrm{H}$ \\
\hline $1 \sigma^{\mathrm{a}}$ & $4 s$ & $1 s$ \\
$2 \sigma^{\mathrm{a}}$ & $4 p$ & $1 s^{\prime}$ \\
$3 \sigma^{\mathrm{a}}$ & $3 d$ & $2 s$ \\
$1 \pi^{\mathrm{a}}$ & & $2 p$ \\
$4 \sigma$ & & \\
$2 \pi$ & & \\
$5 \sigma$ & & \\
\hline \hline
\end{tabular}

a These orbitals were kept doubly occupied in all $n$-electron functions. 
(3) All double excitations from the active orbitals into the Ar atomic orbitals.

(4) All double excitations from the active orbitals into the $\mathrm{H}$ atomic orbitals.

The above discussion of evaluating matrix elements between nonorthogonal functions was couched in terms of SDF's. The four types of configurations shown here give rise to 445 different SDF's, and these would need to be dealt with individually if the simplest version of the theory were used. After applying all the simplifications arising out of the theory of the symmetric groups and the projection of the ${ }^{1} \Sigma^{+}$state, we obtain a $97 \times 97$ NCI matrix. Figure 1 shows the lowest 15 eigenvalues of the Hamiltonian matrix plotted as a function of the internuclear distance. The value of $D_{e}$ from the NCI and the values of some of the asymptotic energies are shown in Table I.

The rank of the matrix of overlaps among the orbitals in a particular Hamiltonian matrix element was another important consideration for evaluating matrix elements. We have only recently modified our program so that it automatically collects statistics on ranks. For this calculation it was found that $53 \%$ of the matrices were nonsingular, $43 \%$ of the matrices had ranks of $n-1$ or $n-2$, and $4 \%$ had rank less than $n-2$. For $\mathrm{ArH}^{+}$the numbers are independent of the internuclear distance. Having a method for calculating the cofactors of singular matrices is therefore essential.

Since the great difficulty of NCI calculations is so frequently commented upon, ${ }^{25}$ we note finally that the present results were all obtained with a Stride Micro 440 (Ref. 26) computer (Reno, Nevada) with 512k memory, 15-Mbyte disk, and a floating-point processor. The calculations averaged about two hours of real time per point. It is clear that immense computer resources are not necessary.

\section{DISCUSSION}

The entrance channel for the scattering experiments that produce $\mathrm{Ar}^{2+}+\mathrm{H}^{-}$corresponds to the lowest adiabatic state in Fig. 1. This is typical of the potential energy curve for two atoms that form a chemical bond. $\mathrm{ArH}^{+}$is isoelectronic with $\mathrm{HCl}$, and we expect very similar bonding characteristics in the two cases. The $D_{e}$ from the NCI is shown in Table I.

The most straightforward method of determining the cross section for 2ECT would involve solving the semiclassical, time-dependent Schrödinger equation for the states shown in Fig. 1. We have so many real states missing from our treatment, however, that a more qualitative discussion seems better justified. This will not give any quantitative confirmation of the experimental results but can give a rationale for the production of $\mathrm{H}^{-}$.

We base our discussion upon an analysis of the wave functions for the various states. The basis functions in the above fourth group of configurations all may be described as having the 2ECT pattern, $\mathrm{Ar}^{2+}+\mathrm{H}^{-}$, at least for the larger internuclear separations. The 2ECT functions span a linear subspace in the whole space of the basis. If this subspace were orthogonal to the subspace of

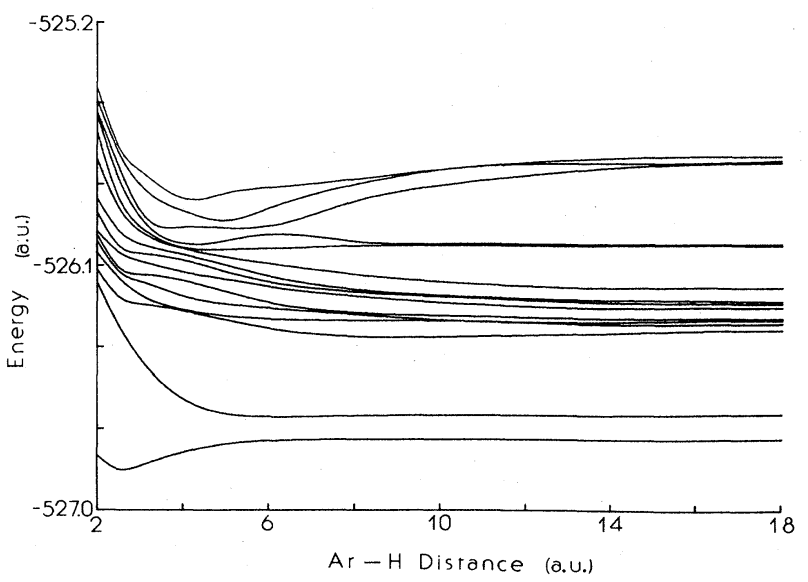

FIG. 1. First 15 adiabatic states for $\mathrm{ArH}^{+}$from the NCI calculation described in text.

the remainder of the basis, then an "occupancy" in any of the eigenvectors of the whole Hamiltonian would be given by an easy calculation and, furthermore, would be a measure of the importance of this physical asymptotic state in the molecular state. In a NCI calculation, of course, this orthogonality is just what is abandoned, and some other method must be devised. There does not seem to be a unique way to define such occupancies, but two methods are given below. As will be argued, these are reasonably expected to bracket the "true physical situation" and, therefore, give bounds useful in qualitative thinking about the superposition of these nonorthogonal states.

Let the nonorthogonal $n$-electron basis of our NCI be symbolized by the functions, $\phi_{i}, i=1,2, \ldots, N$, and let $\phi_{u}$ be a subset of these that represent some physical characteristic we wish to investigate. In this case we have interest in basis functions describing some type of 2ECT. We also let $\phi_{v}$ represent the remainder of the basis functions, i.e., those not having the selected characteristic. If $|\phi\rangle$ represents a row ket of the whole space then the overlap matrix of the basis is

$$
\langle\phi \mid \phi\rangle=\underline{S}=\left[\begin{array}{ll}
\underline{S}_{u u} & \underline{S}_{u v} \\
\underline{S}_{v u} & \underline{S}_{v v}
\end{array}\right],
$$

where the matrix is shown in partitioned form in the second equality. The determinant $|\underline{S}|$ must be nonzero, of course, for the NCI treatment to possess any solution. Therefore, $\underline{S}$ is nonsingular in its entirety, and clearly, $\underline{S}_{u u}$ and $\underline{S}_{v v}$ must also be nonsingular. As is easily seen, the projection operator associated with the $\phi_{u}$ subspace is

$$
F_{u}=F_{u}^{2}=\left|\phi_{u}\right\rangle \underline{S}_{u u}^{-1}\left\langle\phi_{u}\right|,
$$

and its orthogonal complement is $I-F_{u}$. Similarly, the projection operator for the $\phi_{v}$ subspace is

$$
F_{v}=F_{v}^{2}=\left|\phi_{v}\right\rangle \underline{S}_{v v}^{-1}\left\langle\phi_{v}\right|,
$$

and its orthogonal complement is $I-F_{v}$. We note that, in general, $F_{u} \neq I-F_{v}$. These would be equal only if the submatrix $\underline{S}_{u v}=\underline{S}_{v u}^{\dagger}=0$. 
Since $S$ is nonsingular, $\underline{S}^{-1}$ exists, and it may also be written in a partitioned form similar to (11),

$$
\underline{S}^{-1}=\left[\begin{array}{ll}
\left(\underline{S}^{-1}\right)_{u u} & \left(\underline{S}^{-1}\right)_{u v} \\
\left(\underline{S}^{-1}\right)_{v u} & \left(\underline{S}^{-1}\right)_{v v}
\end{array}\right] \text {. }
$$

It will be useful to have explicit expressions for the submatrices of $\underline{S}^{-1}$ in terms of those of $\underline{S}$. The formulas we want are

$$
\begin{aligned}
& \left(\underline{S}^{-1}\right)_{u u}=\left(\underline{S}_{u u}-\underline{S}_{u v} \underline{S}_{v v}^{-1} \underline{S}_{v u}\right)^{-1}, \\
& \left(\underline{S}^{-1}\right)_{v u}=-\underline{S}_{v v}^{-1} \underline{S}_{v u}\left(\underline{S}^{-1}\right)_{u u}, \\
& \left(\underline{S}^{-1}\right)_{v v}=\left(\underline{S}_{v v}-\underline{S}_{v u} \underline{S}_{u u}^{-1} \underline{S}_{u v}\right)^{-1},
\end{aligned}
$$

and a corresponding expression for the other submatrix of each case. That these are correct is easily seen by direct multiplication of $\underline{S}$ and $\underline{S}^{-1}$.

Consider now one of the eigenfunctions of the NCI problem $|\Psi\rangle$,

$$
|\Psi\rangle=|\phi\rangle C=\left|\phi_{u}\right\rangle C_{u}+\left|\phi_{v}\right\rangle C_{v},
$$

where $C$ is the corresponding eigenvector of the Hamiltonian. The eigenvector, too, has been partitioned to match the division of the whole space into subspaces. We may rewrite (18) in two ways as the sum of functions in a subspace and its orthogonal complement,

$$
|\Psi\rangle=\left(I-F_{v}\right)\left|\phi_{u}\right\rangle C_{u}+\left(F_{v}\left|\phi_{u}\right\rangle C_{u}+\left|\phi_{v}\right\rangle C_{v}\right),
$$

and

$$
|\Psi\rangle=\left(\left|\phi_{u}\right\rangle C_{u}+F_{u}\left|\phi_{v}\right\rangle C_{v}\right)+\left(I-F_{u}\right)\left|\phi_{v}\right\rangle C_{v} .
$$

Because of the normalization requirement for $|\Psi\rangle$ we have

$$
1=C_{u}^{\dagger}\left(\underline{S}_{u u} C_{u}+\underline{S}_{u v} C_{v}\right)+C_{v}^{\dagger}\left(\underline{S}_{v u} C_{u}+\underline{S}_{v v} C_{v}\right) .
$$

In light of the two forms of (19), we now modify (20) in two ways to obtain expressions of the form

$$
1=P_{u}+P_{v},
$$

and we have

$$
\begin{aligned}
P_{u a} & =C_{u}^{\dagger}\left\langle\phi_{u}\left|I-F_{v}\right| \phi_{u}\right\rangle C_{u} \\
& =C_{u}^{\dagger}\left[\left(\underline{S}^{-1}\right)_{u u}\right]^{-1} C_{u}, \\
P_{v a} & =1-P_{u a},
\end{aligned}
$$

or

$$
\begin{aligned}
P_{u b} & =1-P_{v b}, \\
P_{v b} & =C_{v}^{\dagger}\left\langle\phi_{v}\left|I-F_{u}\right| \phi_{v}\right\rangle C_{v} \\
& =C_{v}^{\dagger}\left[\left(\underline{S}^{-1}\right)_{v v}\right]^{-1} C_{v} .
\end{aligned}
$$

We may now contrast these two occupation expressions. $P_{u a}$ is calculated from the functions $\left(I-F_{v}\right)\left|\phi_{u}\right\rangle$, which we describe as those portions of the $\phi_{u}$ functions that are unique to the subspace of $F_{u}$ and not duplicated in the subspace of $F_{v}$. In our case, these contain the essence of the physical situation we attribute to the 2ECT states. In any wave function the corresponding $P_{u a}$ quantity can therefore be viewed as a minimal measure of the amount of this physical attribute contained in the wave function.

By way of contrast, $P_{u b}$ is formed from the whole portion of the 2ECT space, not only the functions that nominally have this characteristic. In other words, $P_{u b}$ may be thought of as collecting from the whole space the 2ECT attribute and gives its occupation in $|\Psi\rangle$. Under most circumstances one expects $P_{u b}>P_{u a}$, but this is not an absolute mathematical requirement of the definitions.

Figure 2 shows the range of $P_{u b}$ to $P_{u a}$ as a function of internuclear distance for the ground state, the energy curve leading asymptotically to $\operatorname{Ar}\left[(3 p)^{5} 4 p,{ }^{2} P\right]+\mathbf{H}^{+}$, and the energy curve leading asymptotically to $\mathrm{Ar}^{2+}\left({ }^{1} \mathrm{D}\right)+\mathrm{H}^{-}$. The second of these is typical of $\mathrm{Ar}$ Rydberg states and the third is typical of the 2ECT states. The important point to note is the fairly large 2ECT component in the ground state. This is expected from the conventional Heitler-London picture of bond formation, since for $\mathrm{ArH}^{+}$the 2ECT term is the "ionic" as contrasted to the "covalent" contribution to the bond function. Of course, the terms ionic and covalent must be used metaphorically for $\mathrm{ArH}^{+}$because the overall system is not electrically neutral.

Alternatively, one may rationalize the mixture of these different basis functions in terms of electron correlation. The Heitler-London covalent function overcorrelates the electron motion in the bond and the two ionic terms combine in the total wave functions to reduce this to a more nearly optimal level.

We summarize the sequence of events using a semiclassical, straight-line trajectory description of the collision process. In the entrance channel, as the atom and the ion approach one another at impact parameters less than $\sim 2$ A, a typical chemical bond is temporarily formed between the atoms. Through the action of electron correlation, the bond formation induces into the total wave function a significant contribution from the basis functions of the 2ECT type. Then as the atoms separate after the coilision, this state retains its identity in the diabatic sense,

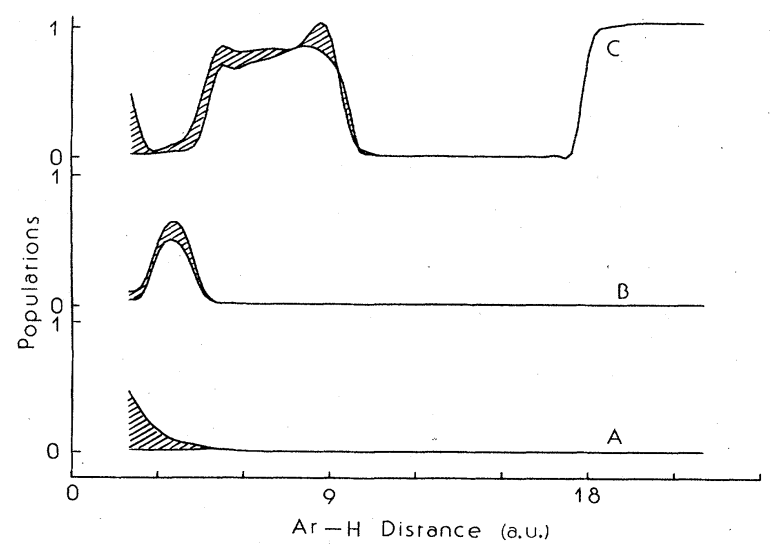

FIG. 2. Range of occupancies of 2ECT subspace for three selected states of calculation. $A$, ground state; $B$, state leading asymptotically to $\operatorname{Ar}\left[(3 p)^{5} 4 p,{ }^{2} P\right]+\mathrm{H}^{+} ;$and $C$, state leading asymptotically to $\mathrm{Ar}^{2+}\left({ }^{1} D\right)+\mathrm{H}^{-}$. 
and some of the total probability appears asymptotically as $\mathrm{Ar}^{2+}$ and $\mathrm{H}^{-}$.

The ground configuration of $\mathrm{Ar}^{2+}$ is $p^{4}$ leading to $L-S$ coupling states of ${ }^{3} P,{ }^{1} D$, and ${ }^{1} S$. The ${ }^{3} P$ state cannot, without a spin flip due to spin-orbit coupling or similar effect, be part of an exit channel with a stable $\mathrm{H}^{-}$. Therefore, two different 2ECT diabatic states, asymptotically connected with $\mathrm{Ar}^{2+}\left({ }^{1} D\right)$ and $\mathrm{Ar}^{2+}\left({ }^{1} S\right)$, thread their way through the adiabatic states as the internuclear distance changes. They show up in the 2ECT occupancy curve [Fig. 2(c)] at two different internuclear separations. The energy for the ${ }^{1} S$ state is higher and appears first (moving outward) at internuclear distances between 5 and 10 bohrs. The lower one appears suddenly around 18 bohrs.

These transitions at larger distances occur very rapidly with changing internuclear distance. At the $2-50-\mathrm{keV}$ energy range of typical experiments, a conventional analysis using the Landau-Zener theory ${ }^{27}$ would therefore be expected to show a low probability for systems remaining on an adiabatic curve as they pass through one of these avoided crossing regions. If the crossings of the 2ECT states with the actual Rydberg and continuum states in this energy range behave in the same way as they do with these discrete CI states, then we expect that the 2ECT diabatic states, once formed, will have a significant probability of existing through the outgoing portion of the trajectory. Those that make it will result in $\mathrm{H}^{-}$ions.

The occupancy curves for the ground state show that the possibility of significant 2ECT state formation occurs inside 4 bohrs, which corresponds to a geometric cross section $\sim 1.4 \times 10^{-15} \mathrm{~cm}^{2}$. From the experimental results we see that, out of 100 collisions with impact parameters $\leq 4$ bohrs, 1 or 2 result in 2 ECT. Therefore, in only a relatively few of the systems need the 2ECT diabatic states survive the trip outward, and the occupancy results from our NCI calculations suggest the trip to be relatively easy.

In the wave functions describing the 2ECT diabatic state the way the $\mathrm{H}^{-}$portion of the system is represented shows a change with internuclear distance. As was discussed above, the orbitals used include both a $1 s$ and $1 s^{\prime}$ atomic orbital centered at the proton. Asymptotically, the $\mathrm{H}^{-}$ion is described principally by the Hylleras-Eckart, split-orbital function, although angular correlation is provided in the basis. Among the $\mathrm{CI}$ functions we have both the $(1 s)^{2}$ and $(1 s)\left(1 s^{\prime}\right)$ configurations represented, ${ }^{28}$ and the relative amounts of these two basis functions in the 2ECT state depend on the internuclear distance. Figure 3 shows a graph of the relative amounts of these basis functions with distance. We see that the $(1 s)^{2}$ configuration is

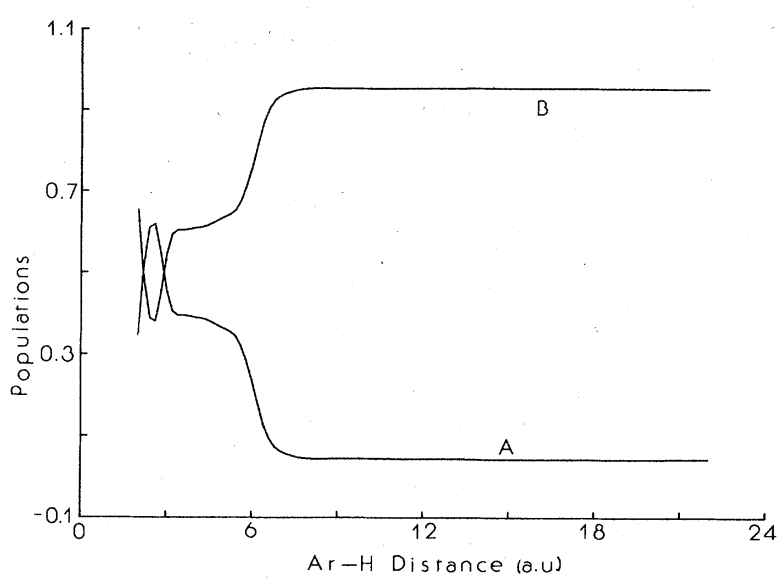

FIG. 3. The relative amounts of the configurations $(1 s)^{2}$ and $(1 s)\left(1 s^{\prime}\right)$ describing the $\mathrm{H}^{-}$ion as a function of distance are shown in the curves $A$ and $B$, respectively.

more important in the bonding region and the $(1 s)\left(1 s^{\prime}\right)$ function becomes more important at long distances. This effect is due principally to the positive charge on the $\mathrm{Ar}^{2+}$ ion; as the $\mathbf{H}^{-}$gets closer to it, the lower energy together with the deeper potential well require the orbitals to have a shorter range. The relatively slow change in the description of the $\mathrm{H}^{-}$portion of the 2ECT states is expected to help in preserving their integrity as the system makes its way outward after the closest approach and to contribute to the $\mathbf{H}^{-}$ion production.

\section{SUMMARY}

We have presented the calculations of some of the lowlying energy curves for the $\mathrm{ArH}^{+}$system using a $\mathrm{NCI}$ procedure described here. This has a novel feature in that basis functions representing both molecular-orbital and valence-bond (Heitler-London) types are included. This provides a compact representation of the wave functions that allows the molecular-orbital configurations to contribute at short distances where they work best and valencebond configurations to contribute asymptotically where they predominate. The wave functions produced are analyzed for the contribution of 2ECT states and the unexpectedly large production of $\mathrm{H}^{-}$during proton on argon collisions is easily rationalized.
${ }^{1}$ Y. M. Fogel, R. V. Mitin, V. G. Kozlov, and N. D. Romashko, Zh. Eksp. Teor. Fiz. 35, 565 (1959) [Sov. Phys._JETP 8, 390 (1959)].

${ }^{2}$ V. V. Afrosimov, R. N. Il'in, and E. S. Soloviev, Zh. Tekh. Fiz. 30, 705 (1960).

3J. F. Williams, Phys. Rev. 150, 7 (1966).

${ }^{4}$ B. Van Zyl, H. L. Rothwell, and H. Neumann, Phys. Rev. A 21, 730 (1980).

${ }^{5}$ B. Van Zyl, M. W. Gealy, and H. Neumann, Phys. Rev. A 33, 2333 (1986).
${ }^{6}$ G. A. Gallup and J. Gerratt (unpublished).

${ }^{7}$ Modern Theoretical Chemistry, edited by H. F. Schaefer III (Plenum, New York, 1977), Vols. 3 and 4.

${ }^{8}$ R. J. Buenker and S. J. Peyerimhoff, in New Horizons of Quantum Chemistry, edited by P.-O. Löwdin and B. Pullman (Reidel, Boston, 1983).

${ }^{9}$ M. Raimondi, W. Campion, and M. Karplus, Mol. Phys. 34, 1483 (1977); M. Raimondi, J. Gerratt, and D. L. Cooper, Phys. Rev. A 34, 673 (1986).

10P.-O. Löwdin, Phys. Rev. 97, 1474 (1955). 
${ }^{11}$ A. C. Aitken, Determinants and Matrices (Interscience, New York, 1956).

${ }^{12}$ H. F. King, R. E. Stanton, H. Kim, R. E. Wyatt, and R. G. Parr, J. Chem. Phys. 47, 1936 (1967).

${ }^{13}$ F. Prosser and S. Hagstrom, Int. J. Quantum Chem. 2, 89 (1968).

${ }^{14}$ G. A. Gallup and J. M. Norbeck, Chem. Phys. Lett. 22, 161 (1973).

${ }^{15}$ G. A. Gallup, R. L. Vance, J. R. Collins, and J. M. Norbeck, Adv. Quantum Chem. 16, 229 (1982), Sec. II D.

${ }^{16}$ In a recent paper, S. C. Leasure and G. G. Balint-Kurti, Phys. Rev. A 31, 2107 (1985), claim to give, for the first time, formulas for evaluating the matrix element (5) that provide an $n^{4}$ algorithm. Their final result [Eq. (47) of this reference] is, however, essentially Löwdin's result [Eqs. (49) and (51) of Ref. 10] restated using the trace formalism, and may be obtained from it in one or two steps. Also see Ref. 9 and G. A. Gallup, Phys. Rev. A 34, 671 (1986).

17P.-O. Löwdin, Phys. Rev. 97, 1509 (1956).

${ }^{18}$ R. Pauncz, Spin Eigenfunctions (Plenum, New York, 1979).

${ }^{19}$ G. A. Gallup, Int. J. Quantum Chem. 21, 1057 (1982).

${ }^{20}$ See, for example, D. E. Rutherford, Substitutional Analysis (Edinburgh University Press, Edinburgh, 1948).

${ }^{21}$ See Ref. 15, all sections.

${ }^{22}$ G. A. Gallup, Int. J. Quantum Chem. 8, 267 (1974).
${ }^{23}$ R. L. Vance and G. A. Gallup, J. Chem. Phys. 73, 894 (1980).

${ }^{24}$ C. Eckart, Phys. Rev. 36, 878 (1930); see also, H. A. Bethe and E. Salpeter, Quantum Mechanics of One- and Two-Electron Atoms (Plenum, New York, 1977), p. 154.

${ }^{25}$ For recent statements see, A. F. Voter and W. A. Goddard, Chem. Phys. 57, 253 (1980); I. D. Petsalakis, G. Theodorakopoulis, C. A. Nicolaides, R. J. Buenker, and S. D. Peyerimhoff, J. Chem. Phys. 81, 3161 (1984); A. D. McLean, B. H. Lengsfield, J. Pacansky, and Y. Ellinger, ibid. 83, 3567 (1985).

${ }^{26}$ The Stride 440 is an MC68000-based computer. Benchmark measurements show it to have a megaflop $\left(10^{6}\right.$ instructions executed per second) rating roughly equivalent to the VAX $11 / 750$. The article by J. J. Dongarra, Technical Memorandum No. 23 (Rev. 2), Argonne National Laboratory (unpublished), provides the data for the comparison.

${ }^{27}$ L. D. Landau, Phys. Z. Sowjetunion 2, 46 (1932); C. Zener, Proc. R. Soc. London, Ser. A 137, 696 (1932).

${ }^{28}$ In this NCI calculation the split-orbital basis for $\mathrm{H}^{-}$is easily dealt with. In an orthogonal CI this same effect would be represented by the equivalent configuration mixing, $\left(1 s^{\prime \prime}\right)^{2}-\lambda(2 s)^{2}$, where $1 s^{\prime \prime}$ is the (unbound) SCF function for $\mathrm{H}^{-}$. The split-orbital representation of the radial correlation is more intuitive, particularly since the SCF function for $\mathrm{H}^{-}$ is quite unphysical. 